\title{
Investigations of Forbush decreases by means of muon hodoscope
}

\author{
N.S. Barbashina", I.I. Astapov, V.V. Borog, A.N. Dmitrieva, R.P. Kokoulin, \\ K.G. Kompaniets, A.A. Petrukhin, O.A. Sitko, V.V. Shutenko, E.I. Yakovleva, \\ I.I. Yashin \\ National Research Nuclear University MEPhI (Moscow Engineering Physics Institute), \\ Moscow 115409, Russian Federation \\ E-mail: NSBarbashina@mephi.ru
}

\begin{abstract}
The results of the analysis of Forbush decreases registered by the muon hodoscope URAGAN in the period from 2006 to 2013 are presented. For the first time, according to the muon hodoscope data, the energy, spatial-angular and temporal characteristics of Forbush decreases were determined using a single setup and a single approach. The dependences of the amplitude of the cosmic ray muon flux intensity decrease on the primary particle energy in the region above 10 $\mathrm{GeV}$ were obtained and their variations at different phases of the Forbush decreases development were studied. Based on the muon snapshot (muonography) analysis, the parameters of the local anisotropy vector value and correlations between its projections to the South and East were investigeted.
\end{abstract}

The 34th International Cosmic Ray Conference

30 July - 6 August, 2015

The Hague, The Netherlands

*Speaker 


\section{Introduction}

Forbush decrease (FD) is a sharp decrease of the cosmic ray (CR) intensity caused by various heliospheric processes. A more general definition of the FD was given in [1]. In that work, authors defined the Forbush decreases as variations of the density and anisotropy of primary cosmic rays caused by large-scale propagating disturbances of the solar wind.

Forbush decreases are observed at different setups registering CR: neutron monitors (NM), muon telescopes, muon hodoscopes, satellite equipments and other detectors (including muon detectors) that are the part of installations for the extensive air shower registration. On the Earth's surface, the Forbush decreases are mainly studied by means of the ground-based neutron monitors. These detectors are sensitive to variations of cosmic rays of relatively low energies, which correspond to large amplitudes of the observed effect. Neutron monitors are combined into a single network which enables a global survey of cosmic ray variations around the Earth using the setups located in different geographical points.

In recent time, the interest in the study of the FD in the cosmic-ray muon flux was significantly increased [2]. This is due to the fact that studies of the FD in the muon flux have several features. Firstly, muons are sensitive to higher (than neutrons) energies of primary cosmic rays (PCR) that allows studying of the heliospheric disturbances responsible for the high-energy PCR modulation. Secondly, which is more important, the muons retain the primary particle directions that enables to obtain spatial-angular picture of the cosmic ray modulations in the near-Earth space and to study their dynamics in a wide range of zenith and azimuth angles using a single setup. These studies became possible due to the creation of the equipment of a new generation - the muon hodoscope [3]. However, the studies of FD in the muon flux have some specifics: the smaller decrease amplitudes of the CR intensity because of the higher primary energy and the necessity to consider the influence of the atmospheric temperature on the muon flux. Such specifics imposes higher requirements to the analysis methods. Therefore, the development of new approaches to the study of FD in the muon flux using the new equipment - muon hodoscope - is a relevant task. Specially for the muon hodoscope, the technique of investigation of the Forbush decreases registered in the hodoscopic mode was elaborated. It was developed on the basis of the analysis of the muon hodoscope URAGAN [4] experimental data for the period from 2006 to 2011. Some results of application of the developed approaches have been published in papers [5-7]. This paper presents the results of the analysis of the FD registered by the URAGAN for a longer period, from 2006 to 2013.

\section{Muon hodoscope URAGAN, experimental data and analyzed events}

Muon hodoscope URAGAN is a modular type wide-aperture setup consisting of four individual supermodules (SM) which operate as autonomous coordinate-tracking detectors. Each SM consists of eight planes of gas-discharge chambers (streamer tubes) equipped with two-coordinate system of external readout strips. Each plane of the SM contains 20 chambers and $320 \mathrm{X}+288 \mathrm{Y}$ registering channels. Such structure ensures rejection of the random triggering, the passage of cascade showers and similar phenomena with a high degree of certainty and reliability. The area of the $\mathrm{SM}$ is $\sim 11.5 \mathrm{~m}^{2}$. The supermodule provides high spatial and angular 
accuracy of muon track reconstruction (correspondingly, $1 \mathrm{~cm}$ and $1^{\circ}$ ) in a wide range of zenith angles from $0^{\circ}$ to $80^{\circ}$. One supermodule of the muon hodoscope URAGAN registers 80 thousand muons per minute. Information about registered muons is stored in two-dimensional angular matrices. This information is a starting material for the further analysis. To solve various problems, the data can be combined both in time and in angular cells, but the initial information is saved. During the FD analysis, the size of the angular cell is 1 by 4 degrees (for the zenithazimuthal grid). To analyze the muon flux variations during the FD, the summed 10-minute data averaged over two SM (for 2006) and over three SM (for 2007 - 2013) corrected for the barometric and temperature effects [8] were used.

During the period from 2006 to 2013, 311 FD were registered according to the URAGAN data. Based on the visual scanning of time series of the URAGAN data, the "complex" FD (the superposition of several FD at phases of the counting rate decrease) were excluded from the further analysis. Only the FD which had the undistorted intervals with a duration of more than one day before and one day after the decrease were selected. A total of 62 FD with amplitudes $\geq 0.5 \%$ were selected. The average value of the FD amplitudes according to the URAGAN data was about 1\%. The maximal FD amplitude - more than 3\% - was registered on March 7, 2012.

For the analysis of the FD energy characteristics, the entire zenith angle range was divided into five intervals with approximately equal statistical reliability: $\left[0^{\circ}-17^{\circ}\right),\left[17^{\circ}-26^{\circ}\right)$, $\left[26^{\circ}-34^{\circ}\right),\left[34^{\circ}-44^{\circ}\right)$, and more than $44^{\circ}$. Studies of the muon flux anisotropy were carried out for the FD selected for the period from 2007 to 2013 (57 FD).

\section{Methods and results of the analysis of the FD energy characteristics}

The URAGAN data are sensitive to the CR energy spectrum index, as the muons registered at different zenith angles are generated by primary particles with different effective energies. To obtain the energy spectrum of amplitudes of Forbush decreases of the muon flux, the standard coupling functions of connection between the primary and secondary CR fluxes were used. Using the collection function $P(E, \theta)$ which determines the number of
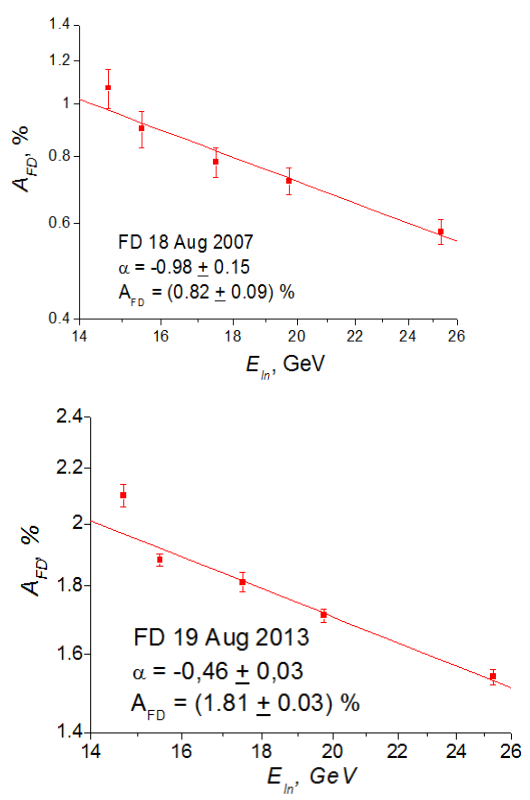
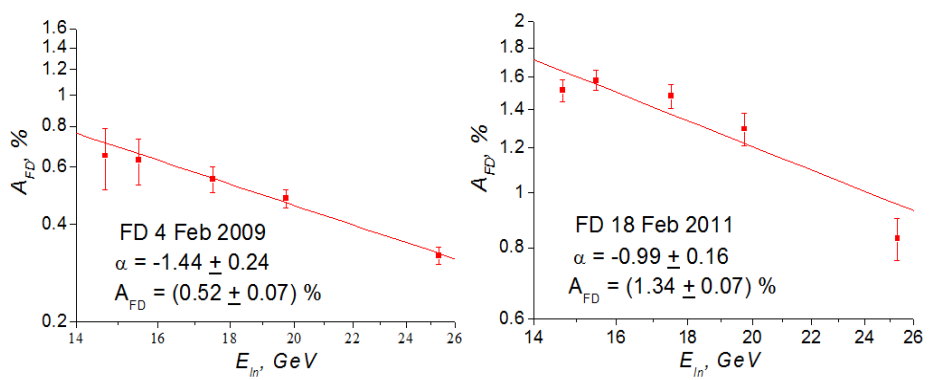

Figure 1: Examples of dependences of the decrease amplitude on the mean logarithmic energy of the primary particles for several FD. 
muons falling within the detector unit aperture (here $E$ is the primary particle energy) and assuming that the PCR flux decrease during the FD is given by $\Delta J_{p} / J_{p}=R^{-\gamma}$ [9], $R$ is the primary particle rigidity, the detector response function $G(E, \theta)$ was calculated. This function gives the dependence of the detector counting rate on the energy of the primary particles at angle $\theta$ and allows to determine the energy range of primary particles which contribute to the detector counting rate decrease.

For five angular ranges, the mean logarithmic energies $\left(E_{l n}\right): 14.7,15.5,17.5,19.7$ and $25.3 \mathrm{GeV}$ [10], correspondingly, were calculated using the function of the detector response to the decrease of the PCR flux intensity. The analysis of the cosmic ray energy spectrum modulations during the FD was conducted on the basis of dependences of $A_{\mathrm{FD}}$ on $E_{l n}$. These dependences were fitted by a power function $E^{\alpha}$. Figure 1 shows the examples of such dependences for several FD.

As seen from the plots presented in Figure 1, the index $\alpha$ takes different values for FD with various amplitudes. Mainly, $\alpha$ is about -1 , but the spectrum can be "softer" (with $\alpha$ about -1.5 ) or "harder" (with $\alpha \sim-0.5$ ). Figure 2 shows the distribution of the obtained estimates of $\alpha$ for 62 FD. This distribution is characterized by the average $\alpha$ value of about -1.00 with the rms-spread of \pm 0.48 .

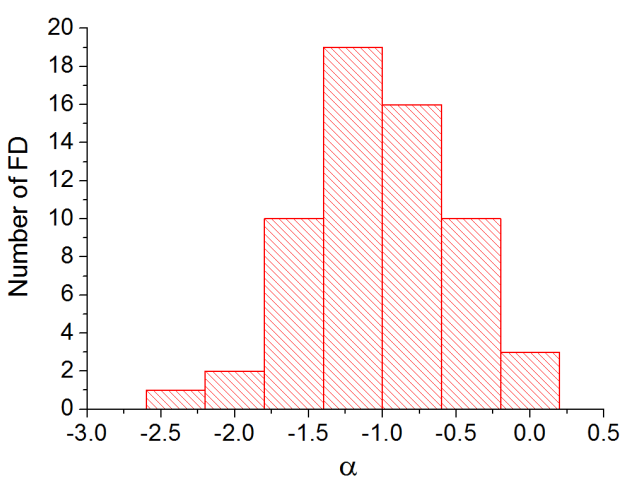

Figure 2: Distribution of the amplitude spectrum indices for $62 \mathrm{FD}$.

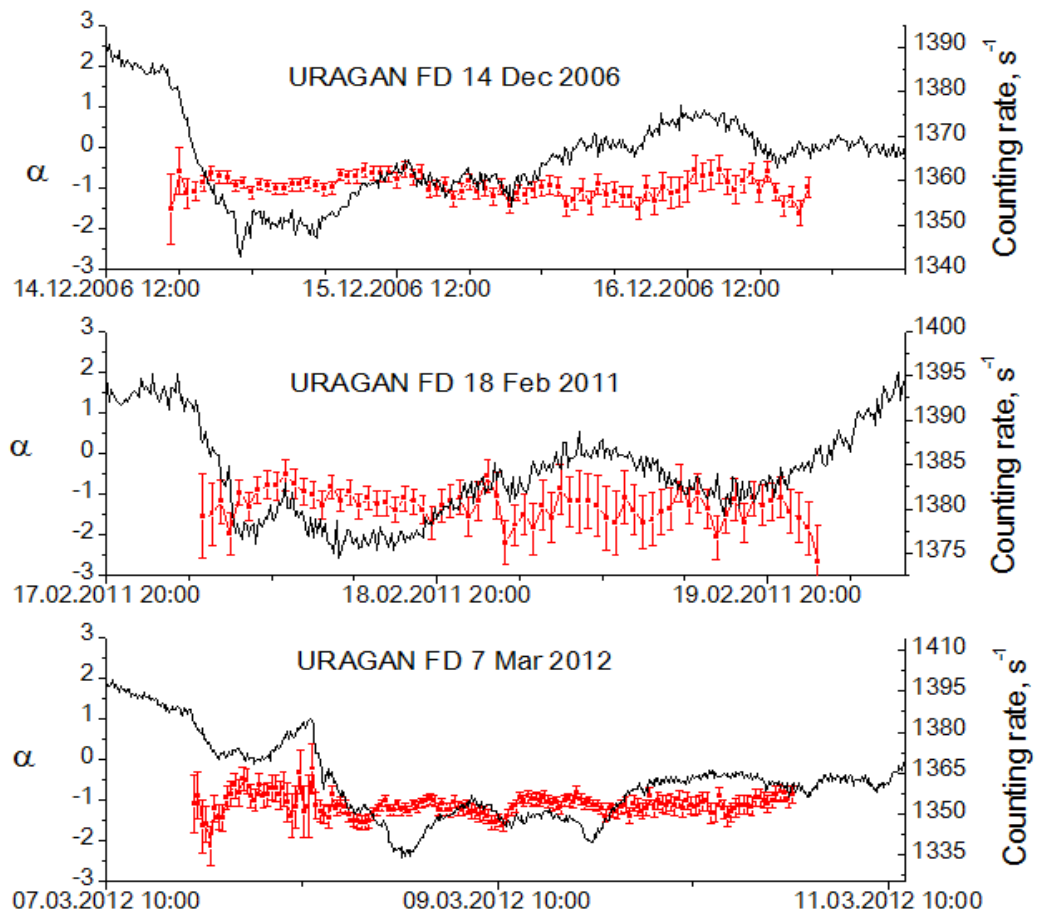

Figure 3: Examples of the behavior of the dynamics of the amplitude spectrum index for the FD on December 14, 2006; February 18, 2011 and March 7, 2012. 
The studies of the temporal dynamics of the amplitude spectrum index $\alpha(\tau)$ at different phases of the event development: decrease, minimum and recovery, which correspond to different phases of the heliospheric disturbance influence on the cosmic ray flux, are of a particular interest. Similar studies were carried out earlier using the data of the neutron monitors and muon telescope [11]. Good temporal and angular resolution of the URAGAN enables to conduct these studies using a single setup. For this purpose, the index of the spectrum decrease was estimated from the URAGAN data in increments of $40-60$ minutes. The decrease amplitudes at different time moments $\left(t_{i}\right)$ were determined as the difference between the daily average counting rate before the decrease and the counting rate averaged for 40 or 60 minutes at the $i$-th step. Figure 3 shows the comparison of the variations of the URAGAN counting rate and $\alpha(\tau)$ for three most powerful FD (December 14, 2006; February 18, 2011 and March 7, 2012).

Figure 4 shows the distribution of the weighted average values of $\alpha(\tau)$ calculated for each of three development phases of 62 FD. The average value of $\alpha(\tau)$ at the decrease is $-0.81 \pm 0.54$, at the phase of minimum $-0.98 \pm 0.53$, and at the phase of recovery $-0.90 \pm 0.65$ (the rms-deviations are indicated). From these distributions, it can be concluded that, in general, no significant changes of $\alpha(\tau)$ at different phases are observed. However, qualitatively one can see that at the phase of the decrease the spectrum of amplitudes FD is harder, at the minimum phase $\alpha \approx-$ 1 , and in the recovery phase the distribution is quite wide, although the average value is of about -1 .

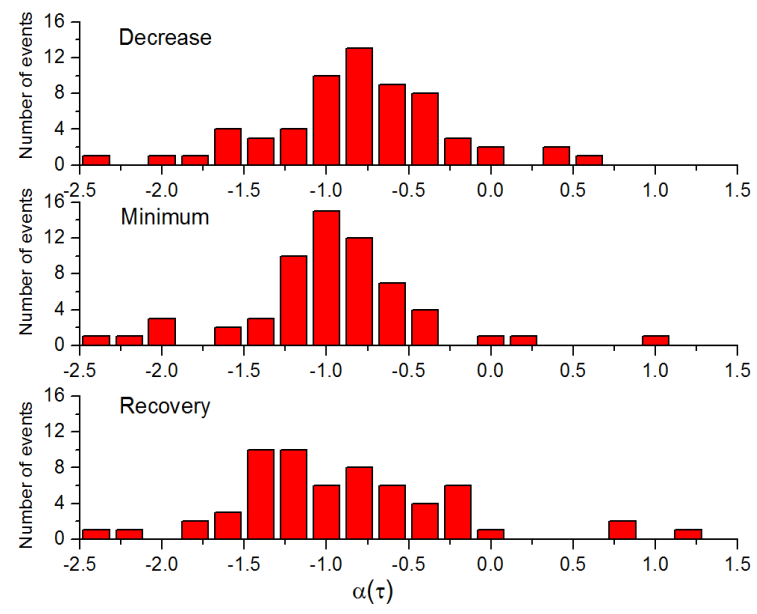

Figure 4: Distribution of the weighted average values of $\alpha(\tau)$ for 62 FD at different phases: decrease, minimum, and recovery.

\section{Methods and results of the analysis of FD according to the muon flux spatial- angular characteristics}

The supermodule response represents the information about triggered strips in each of the two projection planes $X Z$ and $Y Z$. Track parameters (two projection angles) are reconstructed in each projection plane in a real time mode and are accumulated in two-dimensional array $\left(\theta_{X}, \theta_{Y}\right)$ during one-minute interval. Each matrix is normalized to the average matrix obtained by averaging minute matrices for the last day. The result is a matrix, each cell of which contains deviations from the average one in units of their statistical errors. To smooth sharp fluctuation peaks in separate bins, all values of the matrix are smoothed using the Gaussian filter. To optimize the presentation of information considering low statistics in the matrix corners, it is reasonable to present it in the form of a circle limited by zenith angle of $75^{\circ}$. Such data array (matrix) represents a "muon snapshot" (muongraphy) of the upper hemisphere limited by the detector aperture [12]. For 62 FD, the muon snapshots that give visual information about the 
variations of the muon flux angular distribution during the event were plotted. Figure 5 shows the one-hour exposure muon snapshots of the FD of June 23, 2013. Each row of the snapshots in the figure corresponds to the different phase of the FD development: the first row reflects the period before the beginning of the Forbush decrease (23/06/2013 07:00 - 23/06/2013 11:00); the second, phase of the decrease (23/06/2013 12:00 - 23/06/2013 20:00, every 2 hour); then, phase of the minimum (23/06/2013 21:00 - 24/06/2013 09:00, every 3 hours); the last row corresponds to the phase of recovery (24/06/2013 11:00 - 25/06/2013 07:00, every 5 hours).

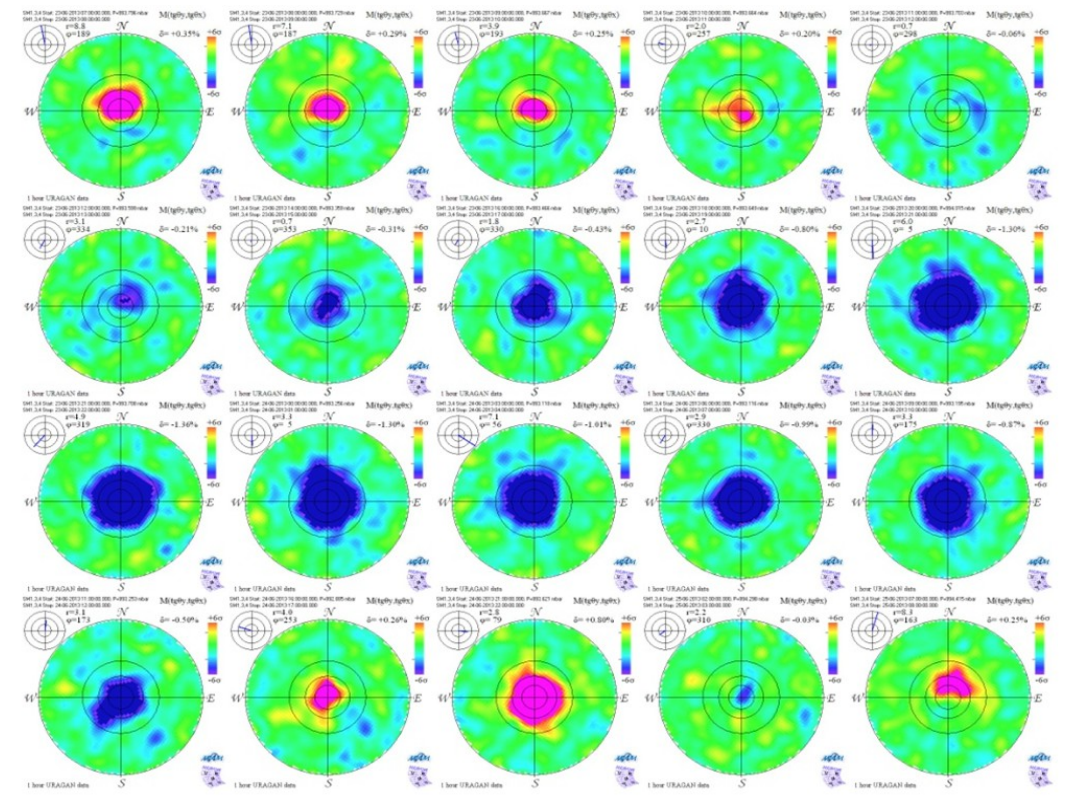

Figure 5: Muon snapshots of the FD of June 23, 2013.
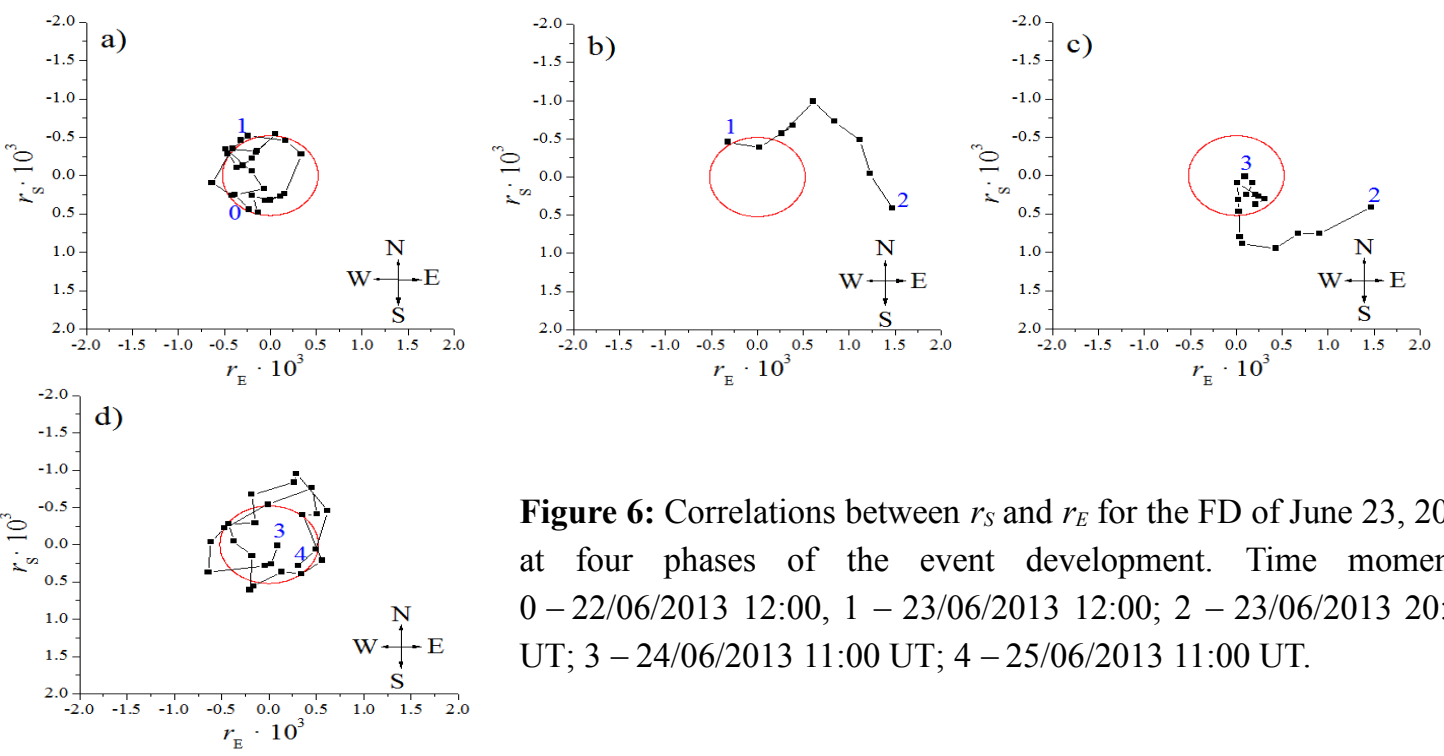

Figure 6: Correlations between $r_{S}$ and $r_{E}$ for the FD of June 23, 2013 at four phases of the event development. Time moments: 0 - 22/06/2013 12:00, 1 - 23/06/2013 12:00; 2 - 23/06/2013 20:00 UT; 3 - 24/06/2013 11:00 UT; 4 - 25/06/2013 11:00 UT.

For a quantitative estimation of these changes, the local anisotropy vector is used. Local anisotropy vector is the sum of unit vectors, each of which has a direction obtained in the reconstruction of a single muon track, normalized to the number of events [13]. The vector can be expanded in directions to the geographic South $\left(A_{S}\right)$ and East $\left(A_{E}\right)$. For the study of deviations from the average anisotropy vector direction, the relative anisotropy vector $\vec{r}$ (a threedimensional vector) and its projection to the South and East are used [14]. The vector $\vec{r}$ 
indicates the muon flux maximum; hence, the minimum is on the opposite side. Its projections $r_{S}$ and $r_{E}$ are calculated in geographic coordinates. Given the primary proton asymptotic trajectories, the geographical direction from North to South at the surface corresponds to the asymptotic direction from East to West, and the geographical direction from West to East corresponds to the asymptotic direction from North to South. The $r_{S}$ and $r_{E}$ quantities are convenient for the study of the muon flux anisotropy variations in the azimuthal directions during the FD [15-17]. Figure 6 shows the correlations between the $r_{S}$ and $r_{E}$ projections.

The circles in the figures correspond to $4 \sigma$ variations of the $r_{S}$ and $r_{E}$ equal to $0.52 \times 10^{-3}$ in a quiet period (from 7 to 10 of February, 2009 which is characterized by the absence of the disturbances in the heliosphere and the Earth's magnetosphere). The exceedance of the circle limits indicates the emergence of a strong anisotropy.
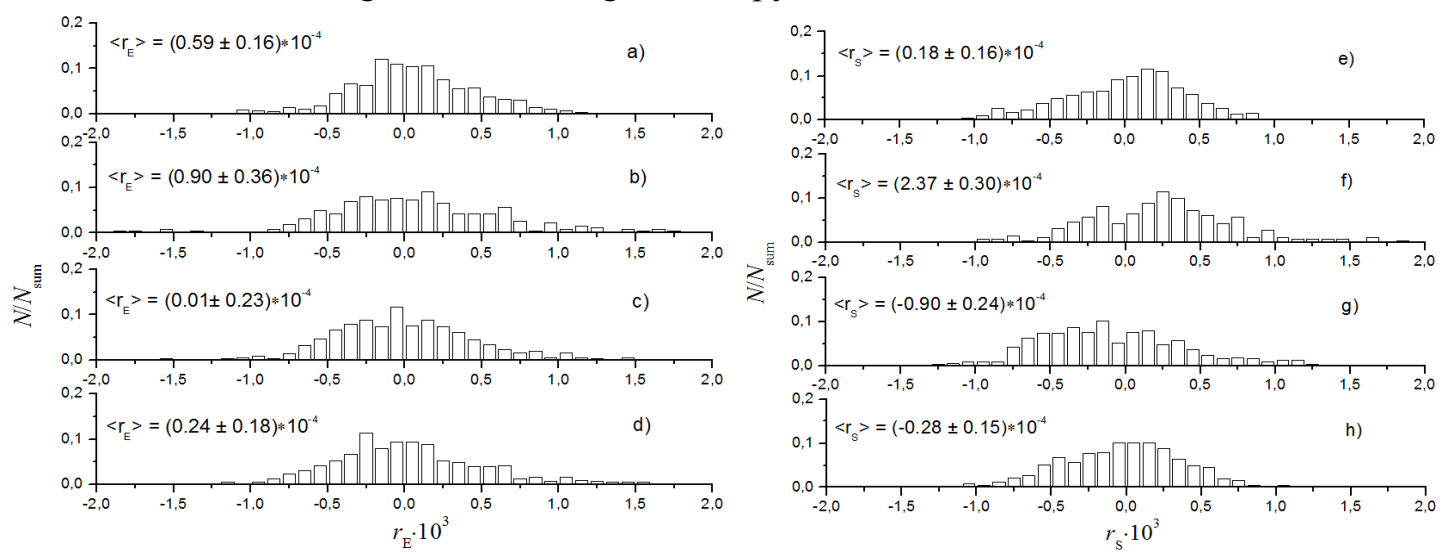

Figure 7: Distribution of values of $r_{E}$ (left) and $r_{S}$ (right) parameters at four FD phases: (a) and (e) before the FD beginning; (b) and (f) - phase of decrease; (c) and (g) - phase of minimum; (d) and (h) phase of recovery.

Such analysis was conducted for 57 FD registed in 2007 - 2013 and showed that the relative anisotropy exceeding $r_{S}$ and $r_{E}$ variations by more than $4 \sigma$ was observed at all phases of $12 \mathrm{FD}$, at three phases of $13 \mathrm{FD}$, at two phases of $12 \mathrm{FD}$, at one phase of $7 \mathrm{FD}$, and was absent at all phases of $13 \mathrm{FD}$. In order to study regularities in the behavior of relative anisotropy in the geographical directions from North to South and from East to West, the distributions of hourly values of the $r_{S}$ and $r_{E}$ parameters for all four phases in which the anisotropy was observed were plotted (Figure 7). These distributions were normalized to the number of points in each phase. As seen in Figure 7, the distributions of the relative anisotropy parameters during the FD for the East-West direction are practically symmetrical. Their mean values (shown in figures with the error of the mean) are close to zero. For the North-South direction (Figure 7, right) at the phase of the decrease the values of $r_{S}$ are clearly shifted to the South and at the phase of the minimum - vice versa. This means that at the phase of dectrase the muon flux minimum was more frequently observed from the North which corresponds to the asymptotic East. At the other FD phases the values of $r_{S}$ remain practically symmetrical.

\section{Conclusion}

Thus, for the first time the integral, energy, spatial-angular and temporal characteristics of the Forbush decreases were determined according to the muon flux variations using a single 
setup and a single approach. The dependences of the amplitudes of decrease of the muon flux intensity on the primary particle energy in the range of $10-30 \mathrm{GeV}$ are in good agreement with the power low character of the energy spectrum of primary cosmic ray modulations with the index $\alpha=-1.00 \pm 0.48$. The analysis of temporal dependences of the indices of the Forbush decrease energy spectra at different phases of their development has shown that indices do not vary significantly depending on the phase. Muongraphies of the FD give a good qualitative description of variations of the muon flux spatial-angular distribution during these events in a real-time mode. Analysis of the projections of the relative anisotropy vector to the South and to the East at different phases of FD development has shown that, on average, at the phase of the counting rate decrease during the FD, the muon flux from the geographic South decreases weaker than from the North, and at the stage of minimum - inversely, which corresponds to the variation of the East-West anisotropy of primary cosmic rays.

\section{Acknowledgements}

The work was performed at the Unique Scientific Facility "Experimental complex NEVOD" with the financial support from the State provided by the Russian Ministry of Education and Science (project No. RFMEFI59114X0002).

\section{References}

[1] A.V. Belov et al., Izv. RAN. Ser. Fiz., 65, 373 (2001), in Russian.

[2] M. Rockenbach et al., Space Sci. Rev. 182, 1 (2014).

[3] V.V. Borog et al., Proc. 24th ICRC, vol. 4, 1291 (1995).

[4] N.S. Barbashina et al., Instrum. Experim. Techn., 51, 180 (2008).

[5] N.S. Barbashina et al., J. Phys.: Conf. Ser., 409, 012189 (2013).

[6] N.S. Barbashina et al., Bull. Russ. Acad. Sci.: Phys., 75, 814 (2011).

[7] N.S. Barbashina et al., Bull. Russ. Acad. Sci. Phys., 73, 343 (2009).

[8] A.N. Dmitrieva et al., Astroparticle Physics, 34, 401 (2011).

[9] L.I. Dorman. Variatsii kosmicheskikh luchei i issledovaniya kosmosa (Cosmic Rays Variations and Space Researches), Moscow: Akad. Nauk SSSR, 1028 (1963), in Russian.

[10] E.I. Yakovleva et al., Bull. Rus. Acad. Sci.: Physics, 73, 357 (2009).

[11] A. Wawrzynczak, M.V. Alania, Adv. Space Res., 45, 622 (2010).

[12] D.A. Timashkov et al., Proc. 30th ICRC, Merida, Mexico, vol. 1, 685 (2007).

[13] V.V. Shutenko et al., Bull. Russ. Acad. Sci. Phys., 73, 347 (2009).

[14] N.S. Barbashina et al., Bull. Russ. Acad. Sci.: Phys., 79, 688 (2015).

[15] V.V. Shutenko et al., Proc. 32nd ICRC (Beijing, China), vol. 11, 276 (2011).

[16] N.S. Barbashina et al., Bull. Russ. Acad. Sci.: Phys., 77, 532 (2013).

[17] V.V. Shutenko et al., Geomagn. Aeronomy, 53, 571 (2013). 